A. Raouf Chouikha (Paris)

\title{
MONOTONICITY OF THE PERIOD FUNCTION FOR SOME PLANAR DIFFERENTIAL SYSTEMS. PART I: CONSERVATIVE AND QUADRATIC SYSTEMS
}

Abstract. We first examine conditions implying monotonicity of the period function for potential systems with a center at 0 (in the whole period annulus). We also present a short comparative survey of the different criteria. We apply these results to quadratic Loud systems $\left(L_{D, F}\right)$ for various values of the parameters $D$ and $F$. In the case of noncritical periods we propose an algorithm to test the monotonicity of the period function for $\left(L_{D, F}\right)$. Our results may be viewed as a contribution to proving (or disproving) a conjecture of Chicone and Jacobs.

1. Introduction. The general question of monotonicity of the period function $T$ has been treated by many authors in the special case when the phase flow has a Hamiltonian form or for special families of vector fields. In particular, this problem has been widely discussed for potential systems

$$
\dot{x}=-y, \quad \dot{y}=g(x),
$$

$(\dot{u}=d u / d t$ ), where different criteria have been given (one may consult $[\mathrm{R}]$, $[\mathrm{Sc}],[\mathrm{Ch}-\mathrm{C}]$ and the references therein).

Following R. Schaaf $[\mathrm{Sc}]$, C. Chicone $[\mathrm{C}]$ points out an especially interesting behavior of the period function for the Neumann boundary value problem $\dot{x}(0)=0, \dot{x}(T)=0$. A solution to this problem corresponds to one of the periodic orbits in the phase plane that starts at time $t=0$ on the $x$-axis and returns to the $x$-axis after time $T$. Monotonicity ensures uniqueness of the solutions with a fixed number of nodes $N$, which correspond to such a periodic trajectory with minimum period $2 T / N$. This situation may occur for autonomous systems other than conservative ones. The interval

2000 Mathematics Subject Classification: 34C15, 34C23, 34C25, 34C37, 37G15.

Key words and phrases: period function, monotonicity, isochronicity, Loud systems, polynomial systems. 
of period values determines the interval of initial conditions for which the equation has a solution.

Another point of interest is the role played by the period function in many perturbation problems (see for example [CLY]). On the other hand, in studying the perturbation of periodic orbits $\gamma$ by Melnikov's method it is assumed that the derivative $T^{\prime}(\gamma)$ is not zero.

For any center 0 of a planar differential system, the largest neighborhood of 0 which is covered by periodic orbits is called the period annulus of 0 and will be denoted by $\gamma_{0}$. A center is said to be a global center when its period annulus is the whole plane. The function $T$ which associates to any periodic orbit $\gamma$ in $\gamma_{0}$ its period is called the period function. The center is called an isochronous center when the period function is constant. A center is said to be nondegenerate when the linearized vector field at the critical point has two nonzero eigenvalues. It is well known that only nondegenerate centers can be isochronous.

When the differential system is analytic, the period annulus of an isochronous center is unbounded.

The orbits may be parametrized for example by choosing their initial values in the segment $(0, \pi)$ on the $x$-axis.

Let $T: \gamma_{0} \rightarrow \mathbb{R}$ be the function defined by associating to every point $(x, 0) \in \gamma_{0}$ the minimum period of a trajectory starting at $(x, 0)$ and reaching the negative $x$-axis. Then $T$ is the period function and is constant on cycles. We say that $T$ is (strictly) increasing if, for every couple of cycles $\gamma_{1}$ and $\gamma_{2}$, with $\gamma_{1}$ included in $\gamma_{2}$, we have $T\left(\gamma_{1}\right) \leq T\left(\gamma_{2}\right)\left(T\left(\gamma_{1}\right)<T\left(\gamma_{2}\right)\right)$.

0 is an isochronous center if $T$ is constant in a neighborhood of 0 .

When the period function is monotone on the whole period annulus we will call it globally monotone.

The interest in isochronicity phenomena arises from several areas. One of them is to better understand the nature of the period function for a family of closed orbits. This in turn is useful in the study of critical periods when analyzing harmonic and subharmonic bifurcations for forced systems.

Apart from this situation and trivial ones, we know very few cases where the behavior of the period function and isochronicity of the center are known. Many more details can be found in the survey article [C-S].

We assume $g(0)=0$, so that the origin is a critical point for the twodimensional problem. Also we suppose $\frac{d g}{d x}(0)=g^{\prime}(0)>0$ and $x g(x)>0$ for $x \neq 0$ so that 0 is a center for (1).

There are many criteria of global monotonicity of period functions for potential systems with a center at 0 . Some of them will be listed below. These conditions are of course related in the sense that some of them imply others. For a complete description and a comparison we refer to [Ch-C]. 
If in addition we suppose $\frac{d^{2} g}{d x^{2}}(0)=g^{\prime \prime}(0)=0$ many global monotonicity conditions reduce to the one of $\mathrm{Z}$. Opial $[\mathrm{O}]$.

Indeed, we first remark that Opial's condition of monotonicity for the period function:

$$
x \frac{d}{d x}\left(\frac{g(x)}{x}\right)>0
$$

is the best among conditions for which $g^{\prime \prime}(0)=0$. Moreover, when $g^{\prime \prime}(0)=0$, Rothe's criterion

$$
R(g)=x\left[3 g^{\prime \prime}(0) g^{\prime}(x)^{2}-g^{\prime \prime}(0) g(x) g^{\prime \prime}(x)-3 g^{\prime}(0)^{2} g^{\prime \prime}(x)\right] \geq 0
$$

reduces to $x g^{\prime \prime}(x) \leq 0$ (which is a particular case of $(\mathrm{Op})$ ).

These results will be used (in Section 3 ) to determine global monotonicity cases of the period function for Loud quadratic systems $\left(L_{D, F}\right)$.

We will describe monotonicity properties of the period function for $\left(L_{D, F}\right)$ when the parameters $D$ or $F$ take one of the critical values

$$
D=0,-\frac{1}{2} \quad \text { and } \quad F=2,1,-\frac{1}{2}, \frac{1}{2} .
$$

In particular, for the systems

$$
\left\{\begin{array}{l}
\dot{x}=-y+x y \\
\dot{y}=x+D x^{2}+2 y^{2}
\end{array}\right.
$$

with a center at the origin we prove the following:

- If $-11 / 10 \leq D<-1 / 2$ the period function decreases in the whole period annulus.

- For $-1 / 2<D<13 / 10$ the period function increases in the whole period annulus.

More generally, a necessary condition for a Loud system $\left(L_{D, F}\right)$ to have a critical period is $F-1+2 D \neq 0$.

Furthermore, [C-J] conjectures that if $\lambda_{3}=\lambda_{6}$ in the Bautin form and the system is not isochronous, then the period function is globally increasing. Our results below may be viewed as a contribution to proving (or disproving) this conjecture.

2. The period function of conservative systems. The results of this part will be useful in what follows. Let us consider the potential system

$$
\dot{x}=-y, \quad y=g(x),
$$

$(\dot{u}=d u / d t)$, where $g \in C^{1}(J, \mathbb{R}), J$ is a real interval containing 0 , and $g(0)=0, g^{\prime}(0)>0$, and $x g(x)>0$ for $x \in J-\{0\}$. So, 0 is a center for (1).

Let $G$ be the primitive of $g$ satisfying $G(0)=0$ and $G(a)=G(b)=c$, with $\alpha<a<0$ and $0<b<\beta,[\alpha, \beta] \subset J$. 
There exists a positive constant $\gamma$ such that $0<c<\gamma$. Notice that the bound $\gamma$ is the energy of the homoclinic orbit enclosing 0 . Then the period function is

$$
T(c)=\sqrt{2} \int_{a}^{b} \frac{d x}{\sqrt{c-G(x)}} .
$$

Let us recall some facts concerning the monotonicity of the period function of the potential system (1). As a corollary we may deduce that when $g$ is at least of class $C^{2}$ Opial's criterion $(\mathrm{Op})$ is optimal among those for which $g^{\prime \prime}(0)=0$ holds.

We first state the following version of a result of Opial [O, Theorem 8]:

Proposition 1. Let $g: J \rightarrow \mathbb{R}$, where $J$ is an interval containing 0 and $g(0)=0$. Suppose $x g(x)>0$ for $x \in J$, and either

(i) $g \in C^{1}(J, \mathbb{R}), g \notin C^{2}(J, \mathbb{R})$, and $g^{\prime}(0)>0$, or

(ii) $g \in C^{2}(J, \mathbb{R}), g^{\prime}(0)>0$ and $g^{\prime \prime}(0)=0$.

Then

$$
\text { if } x \frac{d}{d x}\left(\frac{g(x)}{x}\right)<0 \quad \text { for } x \in J-\{0\},
$$

then the period $T=T(c)$ of (1) is strictly increasing in a neighborhood of 0 ;

$$
\text { if } x \frac{d}{d x}\left(\frac{g(x)}{x}\right)>0 \quad \text { for } x \in J-\{0\},
$$

then the period $T=T(c)$ of (1) is strictly decreasing in a neighborhood of 0 . Moreover, if $g \in C^{3}(J, \mathbb{R}), g^{\prime \prime}(0)=0$ and $g^{(3)}(0) \neq 0$, then $x g^{(3)}(x)$ and $(d / d x)(g(x) / x)$ have the same sign in a neighborhood of 0.

The proof of (2) and (3) may be found for example in [RSC]. For the last part of Proposition 1, consider the function

$$
\psi(x)=x \frac{d}{d x}\left(\frac{g(x)}{x}\right)
$$

obviously of class $C^{2}$. Its derivatives are

$$
\psi^{\prime}(x)=g^{\prime \prime}(x)-\frac{\psi(x)}{x}, \quad \psi^{\prime \prime}(x)=g^{(3)}(x)-\frac{g^{\prime \prime}(x)}{x}+2 \frac{\psi(x)}{x^{2}} .
$$

By the de l'Hospital rule we find

$$
2 \lim _{x \rightarrow 0} \frac{d}{d x}\left(\frac{\psi(x)}{x}\right)=\lim _{x \rightarrow 0} \psi^{\prime}(x)=g^{\prime \prime}(0)=0 .
$$

Again, by the de l'Hospital rule we obtain

$$
\lim _{x \rightarrow 0} \psi^{\prime \prime}(x)=2 \lim _{x \rightarrow 0} \frac{\psi(x)}{x^{2}}=\lim _{x \rightarrow 0} \frac{\psi^{\prime}(x)}{x}=g^{(3)}(0)-\lim _{x \rightarrow 0} \frac{\psi(x)}{x^{2}} .
$$


So, $g^{(3)}(0)$ has the same sign as the function $\psi(x)$. Thus, $\psi(x)$ has a constant sign provided $g^{\prime \prime}(0)=0$.

REMARKS. Let us recall some conditions ensuring the monotonicity of the period function and compare some of them. We adopt the notations of $[\mathrm{Ch}-\mathrm{C}]$.

(i) For $g(x)$ convex in the interval $(a, b)$, S. N. Chow and D. Wang [C-W] stated a criterion of monotonicity for the period function of (1). They proved that

$$
\begin{cases}g^{\prime \prime}(x)>0 & \text { for } x \in(a, b), \\ \Delta(x)=x\left(g^{\prime \prime}(0) g^{\prime}(x)-g^{\prime}(0) g^{\prime \prime}(x)\right) \geq 0(\leq 0) & \text { for } x \in(a, b) .\end{cases}
$$

implies

$$
H_{0}(x)=g(x)^{2}+\frac{g^{\prime \prime}(0)}{3 g^{\prime}(0)^{2}} g(x)^{3}-2 G(x) g^{\prime}(x)>0(<0)
$$

for $x \in(a, b), x \neq 0$, which implies the monotonicity of the period function $T(c)$ for $0<c<\gamma$.

(ii) When $g(x)$ is of class $C^{2}$ and is nonconvex in $(a, b),[\mathrm{C}-\mathrm{W}]$ proved an analogous result, where the function $(d / d x)(g(x) / x)$ in Proposition 1 is replaced by $x\left(d^{2} g / d x^{2}\right)$ (this result is obviously weaker than Proposition 1 because $x\left(d^{2} g / d x^{2}\right)$ and $(d / d x)(g(x) / x)$ share the same sign). However, F. Rothe exhibited a stronger criterion:

$$
R(g)=x\left[3 g^{\prime \prime}(0) g^{\prime}(x)^{2}-g^{\prime \prime}(0) g(x) g^{\prime \prime}(x)-3 g^{\prime}(0)^{2} g^{\prime \prime}(x)\right] \geq 0
$$

for $x \in(a, b)$, which is denoted $f_{4}$ in $[\mathrm{R}]$.

Note that the assumption $x g^{\prime \prime}(x)<0$ for all $x \neq 0$ also implies $\left(\mathcal{C}_{4}\right)$. But then necessarily $g^{\prime \prime}(0)=0$, which is a strong condition (see Proposition 2 below).

(iii) Recall that conditions $\left(\mathcal{C}_{0}\right)$ and $\left(\mathcal{C}_{1}\right)$ were introduced by $[\mathrm{C}-\mathrm{W}]$. The following criterion $\left(\mathcal{C}_{3}\right)$ due to R. Schaaf:

$$
S(g)=5 g^{\prime \prime}(x)^{2}-3 g^{\prime}(x) g^{(3)}(x)>0(<0),
$$

for $x \in(a, b), x \neq 0$, is equivalent to $\left(G^{\prime \prime}\right)^{-2 / 3}$ being convex $(G$ is the primitive of $g$ ).

Let us also recall the criterion $\left(\mathcal{C}_{5}\right)([\mathrm{Ch}-\mathrm{C}])$ which is weaker than $\left(\mathcal{C}_{0}\right)$, and under an additional assumption is more general than the above criteria of Rothe and Schaaf (respectively $\left(\mathcal{C}_{4}\right)$ and $\left(\mathcal{C}_{3}\right)$ ):

$\left(\mathcal{C}_{5}\right) \begin{cases}g^{\prime \prime}(0)\left[3 g^{\prime}(x)^{2}-g(x) g^{\prime \prime}(x)\right]-3 g^{\prime}(0)^{2} g^{\prime \prime}(x) \neq 0 & \text { for } x \in\left(g^{\prime}(0)^{-1}, 0\right), \\ \frac{g^{\prime}(x) g^{\prime \prime}(0)}{g^{\prime \prime}(x) g^{\prime}(0)^{2}} \neq \frac{2 G(x)}{g(x)^{2}} & \text { for } x \in(0, b), x \neq 0 .\end{cases}$ 
It should be noticed that each of these conditions implies that $\left(\mathcal{C}_{0}\right)$ holds, which itself implies the monotonicity of the period.

In the case where the function $g$ is not convex (remark (ii) above), the following proposition underlines relations between different monotonicity conditions for the period function. Even if this result is not new, at least it has the advantage of making precise some facts of [R, Theorem 1]. However, especially in the case where $g(x)$ is of class $C^{2}$ or $C^{3}$, we may notice that the condition $g^{\prime \prime}(0)=0$ appears to be very restrictive. This emphasizes the importance of Rothe's criterion, which avoids that condition.

Proposition 2. Suppose the function $g(x)$ is of class $C^{2}$ in an interval $J$ containing 0 and satisfies $x g(x)>0$ for $x \in J$ and $g^{\prime}(0)>0$. Let $G(x)$ be the primitive of $g(x)$ satisfying $G(0)=0$ and $G(a)=G(b)=c, \alpha<a<0<$ $b<\beta,[\alpha, \beta] \subset J$. Then the following implications hold for any $x \in[a, b]$ :

$$
x g^{\prime \prime}(x)<0 \Rightarrow g(x)^{2}-2 G(x) g^{\prime}(x)>0 \Rightarrow x \frac{d}{d x}\left(\frac{g(x)}{x}\right)<0,
$$

provided that $g^{\prime \prime}(0)=0$. Moreover, each of these conditions implies that the period function $T(c)$ of (1) is strictly increasing for $0<c<\gamma$.

$$
x g^{\prime \prime}(x)>0 \Rightarrow g(x)^{2}-2 G(x) g^{\prime}(x)<0 \Rightarrow x \frac{d}{d x}\left(\frac{g(x)}{x}\right)>0,
$$

provided that $g^{\prime \prime}(0)=0$. Moreover, each of these conditions implies that $T(c)$ is strictly decreasing for $0<c<\gamma$.

If we suppose in addition $g(x)$ is of class $C^{3}$, then

$$
g^{(3)}(x)<0(>0) \quad \text { and } \quad g^{\prime \prime}(0)=0 \Rightarrow x g^{\prime \prime}(x)<0(>0) .
$$

Proof. Notice that, since $G(x)>0$ for $x \neq 0$ and

$$
\frac{d}{d x}\left[g(x)^{2}-2 G(x) g^{\prime}(x)\right]=-2 G(x) g^{\prime \prime}(x),
$$

it follows that $2 x G(x) g^{\prime \prime}(x)<0$ (resp. $>0$ ) implies $g(x)^{2}-2 G(x) g^{\prime}(x)>0$ $($ resp. $<0)$, because $x(d / d x)\left[g(x)^{2}-2 G(x) g^{\prime}(x)\right]$ and $-x g^{\prime \prime}(x)$ have the same sign. So, the first two implications are proved.

The second implication, $g(x)^{2}-2 G(x) g^{\prime}(x)>0$ (resp. $\left.<0\right)$ implies $x(d / d x)(g(x) / x)<0$ (resp. $>0$ ), has been proved by Rothe (see [R, Proposition 4, p. 138]; according to his notations: $\left.h_{3}^{+} \subset h_{2}^{+}\right)$.

Note that the conditions

$$
x g^{\prime \prime}(x)<0(\text { resp. }>0) \text { and } g(x)^{2}-2 G(x) g^{\prime}(x)>0(\text { resp. }<0)
$$

independently imply

$$
H_{0}(x)=g(x)^{2}+\frac{g^{\prime \prime}(0)}{3 g^{\prime}(0)^{2}} g(x)^{3}-2 G(x) g^{\prime}(x) \geq 0(\text { resp. } \leq 0)
$$


and imply that the period function is increasing (resp. decreasing); see [C-W, Corollary 2.3].

On the other hand, the condition $g^{(3)}(x)<0(>0)$ and $g^{\prime \prime}(0)=0$ implies that

$$
H_{3}(x)=5 g^{\prime \prime}(x)^{2}-3 g^{\prime}(x) g^{(3)}(x)>0(<0) \quad \text { for } x \in(a, b), x \neq 0,
$$

which in turn implies $\left(\mathcal{C}_{0}\right)$.

By Proposition 1, $g^{(3)}(0)$ has the same sign as the function $\psi(x)=$ $x(d / d x)(g(x) / x)$. Thus, $g^{(3)}(x)<0(>0)$ and $g^{\prime \prime}(0)=0$ implies $x g^{\prime \prime}(x)<0$ $(>0)$, which implies $x(d / d x)(g / x)<0(>0)$.

From the latter remark, if $g^{(3)}(0) \neq 0$ the function $x(d / d x)(g(x) / x)$ has the same sign as $g^{(3)}(0)$.

3. An application to quadratic systems. We will apply the preceding results to prove some global monotonicity (or nonmonotonicity) cases of the period function for some quadratic systems with a center at the origin. This allows one in particular to study the bifurcation of critical periods from their centers.

It is well known [C-J] that any quadratic system with a center at the origin may be transformed under a linear change of coordinates to a Bautin form:

$$
\left(B_{\lambda}\right) \quad\left\{\begin{array}{l}
\dot{x}=-y-\lambda_{3} x^{2}+\left(2 \lambda_{2}+\lambda_{5}\right) x y+\lambda_{6} y^{2} \\
\dot{y}=x+\lambda_{2} x^{2}+\left(2 \lambda_{3}+\lambda_{4}\right) x y-\lambda_{2} y^{2}
\end{array}\right.
$$

After a rotation of coordinates, the Bautin system $\left(B_{\lambda}\right)$ may be transformed to the Loud system

$$
\left(L_{B, D, F}\right) \quad\left\{\begin{array}{l}
\dot{x}=-y+B x y, \\
\dot{y}=x+D x^{2}+F y^{2},
\end{array}\right.
$$

for some real parameters $B, D$ and $F$. Notice that if $B \neq 0$, then another change of variables $u=B x$ and $v=B y$ brings the preceding system to the form

$$
\left(L_{D, F}\right) \quad\left\{\begin{array}{l}
\dot{x}=-y+x y, \\
\dot{y}=x+D x^{2}+F y^{2},
\end{array}\right.
$$

called a dehomogenized Loud system.

So, for studying the monotonicity of the period function for quadratic systems it suffices the consider their reduced forms $\left(L_{D, F}\right)$.

Recall that the quadratic system (of Bautin form) has an isochronous center at 0 if and only if either it is linear or by rotation of the axes it can be transformed into a Loud system with $(D, F)$ being one of

$$
(0,1), \quad(-1 / 2,2), \quad(0,1 / 4), \quad(-1 / 2,1 / 2) \text {. }
$$

Each of these quadratic isochronous systems has a period annulus that extends from the origin to a separatrix orbit on the Poincaré sphere. Note that 
at most two critical periods bifurcate from an isochronous center in a Loud system.

More precisely, Chicone and Jacobs [C-J] proved that a quadratic isochronous system under a quadratic perturbation of the form

$$
\left\{\begin{array}{l}
\dot{x}=\sum_{1 \leq i+j \leq 2}\left[a_{i j}+O(\varepsilon)\right] x^{i} y^{j}, \\
\dot{y}=\sum_{1 \leq i+j \leq 2}\left[b_{i j}+O(\varepsilon)\right] x^{i} y^{j},
\end{array}\right.
$$

separates at most one family of limit cycles for $\left(L_{-1 / 2,1 / 2}\right)$ when $\varepsilon \neq 0$ and at most two families of limit cycles for $\left(L_{0,1}\right),\left(L_{0,1 / 4}\right)$, or $\left(L_{-1 / 2,2}\right)$.

We will describe the monotonicity properties of the period function for some systems $\left(L_{D, F}\right)$ when the parameters $D$ and $F$ take one of the critical values

$$
D=0,-\frac{1}{2} \text { and } F=2,1,-\frac{1}{2}, \frac{1}{2} .
$$

A general description of our method to determine the monotonicity cases of the period function for any Loud system $\left(L_{D, F}\right)$ will be given in an appendix at the end of the paper.

Furthermore, $[\mathrm{C}-\mathrm{J}]$ conjectures that if $\lambda_{3}=\lambda_{6}$ in the Bautin form and the system is not isochronous, then the period function is globally increasing. Our results below may be viewed as a contribution to proving (or disproving) this stronger conjecture.

3.1. Analysis of Loud systems $\left(L_{D, 2}\right)$. First consider systems corresponding to $F=2$ with a center at 0 ,

$$
\left(L_{D, 2}\right) \quad\left\{\begin{array}{l}
\dot{x}=-y+x y \\
\dot{y}=x+D x^{2}+2 y^{2}
\end{array}\right.
$$

It is known $[\mathrm{L}]$ that if $(1+2 D)(7+5 D) \neq 0$ the period function $T(\gamma)$ of $\left(L_{D, 2}\right)$ is increasing in a neighborhood of 0 .

As we will see, it seems that for $D \neq-1 / 2$ and $D>-7 / 5$ the period function is globally monotonic in the whole period annulus, but we cannot prove it.

To investigate this, we will change the system $\left(L_{D, 2}\right)$ to a potential one, and we will apply different criteria described in Section 2.

More precisely, we prove the following

Theorem 3. Consider a Loud system $\left(L_{D, 2}\right)$ with a center at 0 . Then:

- If $-11 / 10 \leq D<-1 / 2$ then the period function decreases in the whole period annulus. 
- If $-1 / 2<D \leq 13 / 10$ then the period function increases in the whole period annulus.

The system $\left(L_{-1 / 2,2}\right)$ has an isochronous center at 0 .

Proof. Following Loud [L], we will use the following transformation of the system $\left(L_{D, 2}\right)$ :

$$
\begin{aligned}
& x=1-(1+2 u)^{-1 / 2} \equiv \phi(u), \\
& y=\frac{\dot{x}}{x-1}=\frac{\phi^{\prime}(u) \dot{u}}{\phi(u)-1} .
\end{aligned}
$$

One obtains the scalar equation

$$
\text { (E) } \ddot{u}+g(u)=0
$$

where

$$
g(u)=D+(1+D)(1+2 u)-(1+2 D)(1+2 u)^{1 / 2} .
$$

Let $\gamma$ be a periodic orbit of the Loud system which surrounds the origin. The interior of $\gamma$ does not contain a critical point other than 0 . The critical points of $\left(L_{D, 2}\right)$ are $\left(u_{0}, y_{0}\right)$ such that $g\left(u_{0}\right)=0$ and $y_{0}=0$. The line $x=1$ is invariant. So, $\gamma$ cannot intersect this line. Thus, the system

$$
\frac{d u}{d t}=y, \quad \frac{d v}{d t}=-g(u)
$$

which is equivalent to $(E)$ has periodic orbits $\bar{\gamma}$ surrounding the origin such that $-1 / 2<u$ and $u g(u)>0$ for $u \neq 0$. Moreover, $u$ lies in the strip $\alpha<u<\beta$ where $-1 / 2<\alpha<0<\beta$.

Any periodic orbit of equation $(E)$ must lie in the half-plane $1+2 u>0$. Hence, the corresponding orbit of the system $\left(L_{D, 2}\right)$ has the same period $T$. Let $\gamma$ and $\gamma^{\prime}$ be two periodic orbits of $\left(L_{D, 2}\right)$ such that $\gamma \subset \gamma^{\prime}$. Then the corresponding orbits of $(E), \bar{\gamma}$ and $\overline{\gamma^{\prime}}$, are such that $\bar{\gamma} \subset \overline{\gamma^{\prime}}$. Thus,

$$
T(\bar{\gamma})=T(\gamma) \leq(\text { resp. } \geq) T\left(\overline{\gamma^{\prime}}\right)=T\left(\gamma^{\prime}\right)
$$

means that the period function is increasing (resp. decreasing).

A calculation yields

$$
\begin{aligned}
g^{\prime}(u) & =2(1+D)-(1+2 D)(1+2 u)^{-1 / 2}, \\
g^{\prime \prime}(u) & =(1+2 D)(1+2 u)^{-3 / 2}, \\
g^{(3)}(u) & =-3(1+2 D)(1+2 u)^{-5 / 2} .
\end{aligned}
$$

Schaaf's monotonicity condition $\left(\left(\mathcal{C}_{3}\right)\right.$ in Section 2$)$ asserts that if

$$
S(g)=5 g^{\prime \prime}(u)^{2}-3 g^{\prime}(u) g^{(3)}(u)<0
$$

then the period function of $(E)$ decreases. In our case

$$
\begin{aligned}
S(g)= & 5(1+2 D)^{2}(1+2 u)^{-3} \\
& +9(1+2 D)(1+2 u)^{-5 / 2}\left[2(1+D)-(1+2 D)(1+2 u)^{-1 / 2}\right] .
\end{aligned}
$$


After simplifying one gets

$$
S(g)=18(1+D)(1+2 D)(1+2 u)^{-5 / 2}-4(1+2 D)^{2}(1+2 u)^{-3} .
$$

Thus $-1 \leq D<-1 / 2$ implies the period function is decreasing.

Furthermore, since Rothe's monotonicity condition $\left(\mathcal{C}_{4}\right)$ is better than Schaaf's $\left(\mathcal{C}_{2}\right)$, one can hope for an improvement while enlarging the interval of monotonicity of the period function.

More precisely, the Rothe function

$$
R(g)=3 g^{\prime \prime}(0) g^{\prime}(u)^{2}-g^{\prime \prime}(0) g(u) g^{\prime \prime}(u)-3 g^{\prime}(0)^{2} g^{\prime \prime}(u)
$$

yields

$$
\begin{aligned}
R(g)= & 3(1+2 D)\left[2(1+D)-(1+2 D)(1+2 u)^{-1 / 2}\right]^{2} \\
& -(1+2 D)^{2}(1+2 u)^{-3 / 2}[D+(1+D)(1+2 u) \\
& \left.-(1+2 D)(1+2 u)^{1 / 2}-3(1+2 D)(1+2 u)^{-3 / 2}\right] .
\end{aligned}
$$

After simplifying this gives

$$
\begin{aligned}
R(g)= & (1+2 D)\left[X^{1 / 2}-1\right] \\
& \times\left[-\left(2 D^{2}+D+3\right) X^{-1}-13(1+D)(1+2 D) X^{-1 / 2}+12(1+D)^{2}\right]
\end{aligned}
$$

where $X=1+2 u$. An easy calculation shows that if $-11 / 10<D<13 / 10$ then $100 D^{2}-20 D-143$ has no real square root. We deduce that $u=0$ is the only root of $R(g)=0$. Thus, according to Rothe's criterion, $u R(g)(u)>0$ implies the period function is decreasing.

On the other hand, Chicone and Dumortier [C-D] showed that the period function may have a critical point when $D<-7 / 5$ and $F=2$. Thus $T$ cannot be monotonic.

Perhaps we may improve the lower bound of $D$ from $-11 / 10$ down to $-7 / 5$ so that the period function of the corresponding system increases. Note that the Rothe criterion fails for $\left(L_{-7 / 5,2}\right)$.

The Rothe function takes the following form:

$$
R(u)=\frac{1242}{125}\left(X^{-1 / 2}-1\right)^{2}\left[X^{-1 / 2}-\frac{8}{23}\right]
$$

where $X=1+2 u$.

The period function $T$ for a general Loud system has the expression [L]

$$
T(\gamma)=2 \pi+\frac{\pi}{12}\left[10 D^{2}+10 D F+4 F^{2}-D-5 F+1\right] \xi^{2}+o\left(\xi^{2}\right)
$$

(the orbit $\gamma$ starting from the point $(\xi, 0)$ at $t=0$ ). We may deduce that $T$ is increasing near zero if

$$
5 g^{\prime \prime}(0)^{2}-3 g^{\prime}(0) g^{(3)}(0)=10 D^{2}+10 D F+4 F^{2}-D-5 F+1>0 .
$$


For the system $\left(L_{D, 2}\right)$ this reads

$$
5 g^{\prime \prime}(0)^{2}-3 g^{\prime}(0) g^{(3)}(0)=2(1+2 D)(7+5 D)>0 .
$$

Remarks. (a) As a consequence we deduce a result of [F-G-G; Prop. 19]. They proved that the system

$$
\left\{\begin{array}{l}
\dot{x}=-y+\delta x y \\
\dot{y}=x+2 \delta x^{2}+\delta y^{2}
\end{array}\right.
$$

has a decreasing period function. By an easy change of variables one obtains

$$
\left(L_{-1,2}\right) \quad\left\{\begin{array}{l}
\dot{x}=-y+x y \\
\dot{y}=x-x^{2}+2 y^{2}
\end{array}\right.
$$

which is contained in the above family.

(b) It seems that Rothe's criterion for the monotonicity of the period of $(E)$ fails when $D \notin[-11 / 10,-1 / 2]$. It will be interesting to improve Theorem 3 for example by using more powerful criteria than the one given in the preceding section. However, we hope that for the system $\left(L_{D, 2}\right)$ the period function $T$ is decreasing when $D \in[-7 / 5,-11 / 10]$.

(c) Monotonicity conditions for the period function may also be applied to systems other than potential or Loud systems. For example, for Hamiltonian systems of the form $H(x, y)=F(x)+G(y)$ with a center at the origin the monotonicity of the period function has been studied by A. Cima, A. Gasull and F. Mañosas (Sect. 6 in [CGM]).

They proved that if $\psi_{F}(h)>0$ and $\psi_{G}(h)>0$ where

$$
\psi_{F}(h)=\frac{1}{2}\left[\phi_{F}\left(x^{+}\right)-\phi_{F}\left(x^{-}\right)\right] \quad \text { and } \quad \phi_{F}(x)=\frac{1}{2} \frac{F^{\prime}(x)^{2}-2 F(x) F^{\prime \prime}(x)}{F^{\prime}(x)^{3}},
$$

then the period function $T(h)$ is increasing.

Therefore, if $\phi_{F}(x)$ is monotone then $\psi_{F}(h)>0$ implying $T(h)$ is increasing. This criterion of monotonicity may be improved.

Indeed, the hypothesis of $\phi_{F}(x)$ monotone which implies $\phi_{F}(x)>\phi_{F}(0)$ also implies $\psi_{F}(h)>0$. To see that, by the de l'Hospital rule one obtains

$$
\phi_{F}(0)=\lim _{x \rightarrow 0} \phi_{F}(x)=-\frac{1}{6} \frac{F^{(3)}(0)}{F^{\prime \prime}(0)^{2}}
$$

and $\phi_{F}(x)>\phi_{F}(0)$ yields $\phi_{F}\left(x^{+}\right)>\phi_{F}(0)>\phi_{F}\left(x^{-}\right)$.

Thus, the condition $\phi_{F}(x)>\phi_{F}(0)$ is stronger than $\phi_{F}(x)$ monotone. These two criteria ensure the monotonicity of the period function.

3.2. Other Loud systems $\left(L_{D, F}\right)$. In this section we will use different criteria described in Section 2 (essentially those of Schaaf and Rothe). Various cases of monotonicity for the period function of Loud systems $\left(L_{D, F}\right)$ with a center at the origin will be proved. 
3.2.1. Systems $\left(L_{(1-F) / 2, F}\right)$ for any real $F$. Consider first systems $\left(L_{D, F}\right)$ where $D=(1-F) / 2$. We shall prove that if $F-1+2 D=0$ then the period function of this system is monotonic. Opial's condition

$$
u g^{\prime \prime}(u) \neq 0
$$

ensures the monotonicity of the period function for the potential system $u^{\prime \prime}+g(u)=0$ with a center at 0 when $g^{\prime \prime}(0)=0$. Recall that the Rothe function $R(g)=3 g^{\prime \prime}(0) g^{\prime}(u)^{2}-g^{\prime \prime}(0) g(u) g^{\prime \prime}(u)-3 g^{\prime}(0)^{2} g^{\prime \prime}(u)$ reduces to Opial's when $g^{\prime \prime}(0)=0$.

We then prove the following

THEOREM 4. Consider the following Loud system with a center at 0 :

$$
\left(L_{(1-F) / 2, F}\right) \quad\left\{\begin{array}{l}
\dot{x}=-y+x y \\
\dot{y}=x+\frac{1-F}{2} x^{2}+F y^{2} .
\end{array}\right.
$$

Then:

(a) If $1<F<2$ the period function decreases in the whole period annulus.

(b) If $F<1$ or $F>2$ the period function increases in the whole period annulus.

(c) If $F=1$ or $F=2$ the origin is an isochronous center.

Proof. We proceed as in the proof of Theorem 3. Notice that a periodic orbit of the above system which surrounds the origin must lie in the half plane $1-x>0$ since $\dot{x}=d x / d t=0$ on the bounding line $x=1$.

Following Loud [L], the system $\left(L_{(1-F) / 2, F}\right)$ may be transformed to a second order equation by means of the substitution

$$
\begin{aligned}
& x=1-(1+F u)^{-1 / F} \equiv \phi(u), \\
& y=\frac{\dot{x}}{x-1}=\frac{\phi^{\prime}(u) \dot{u}}{\phi(u)-1} .
\end{aligned}
$$

After this transformation one obtains the potential equation

$$
\text { (E) } \ddot{u}+g(u)=0
$$

where

$$
g(u)=\frac{3-F}{2}(1+F u)-(2-F)(1+F u)^{1-1 / F}+\frac{1-F}{2}(1+F u)^{1-2 / F} .
$$

By the remark above the orbits of equation $(E)$ must lie in the half-plane $1+F u>0$. Moreover, by the above transformation equation $(E)$ and system $\left(L_{(1-F) / 2, F}\right)$ have the same period function $T$. A calculation yields

$$
\begin{gathered}
g^{\prime}(0)=1, \quad g^{\prime \prime}(0)=0, \\
u g^{\prime \prime}(u)=(F-1)(2-F) u(1+F u)^{2-2 / F}\left[(1+F u)^{1 / F}-1\right],
\end{gathered}
$$


and

$g^{(3)}(u)=\left(1-F^{2}\right)(2-F)(1+F u)^{-2-1 / F}+(1-F)\left(F^{2}-4\right)(1+F u)^{-2-2 / F}$.

So, $u g^{\prime \prime}(u)$ has the same sign as the product $(F-1)(2-F)$. Then by Proposition 2 one gets (a) and (b) of our theorem. Assertion (c) is due to Loud [L].

3.2.2. Systems $\left(L_{D,-1}\right)$. The following gives increasing cases of the period for systems coresponding to $F=-1$ and any parameter $D$. Consider the quadratic system

$$
\left(L_{D,-1}\right) \quad\left\{\begin{array}{l}
\dot{x}=-y+x y \\
\dot{y}=x+D x^{2}-y^{2} .
\end{array}\right.
$$

THEOREM 5. For the system $\left(L_{D,-1}\right)$ with a center at 0 , the period function increases in the whole period annulus for any value of $D$.

Proof. The function $g(u)$ of the corresponding potential system has the expression

$g(u)=(1-u)\left[(1+D)-(1+2 D)(1-u)+D(1-u)^{2}\right]=-D u^{3}+(D-1) u^{2}+u$.

Its successive derivatives are

$$
g^{\prime}(u)=-3 D u^{2}+2(D-1) u, \quad g^{\prime \prime}(u)=-6 D u+2(D-1) .
$$

The Schaaf function may be written as

$$
\begin{aligned}
5 g^{\prime \prime}(u)^{2}-3 g^{\prime}(u) & g^{(3)}(u) \\
& =5[2(D-1)-6 D u]^{2}+18 D\left[1+2(D-1) u-3 D u^{2}\right] \\
& =126 D^{2} u^{2}-84 D(D-1) u+20(D-1)^{2}+18 D \\
& =126\left[D u-\frac{D-1}{3}\right]^{2}+9\left[D^{2}-D+1\right]>0
\end{aligned}
$$

for any $D$.

3.2.3. Systems $\left(L_{-1 / 2, F}\right)$

Theorem 6. Consider the system $\left(L_{-1 / 2, F}\right)$ with a center at 0 ,

$$
\left(L_{-1 / 2, F}\right) \quad\left\{\begin{array}{l}
\dot{x}=-y+x y, \\
\dot{y}=x-\frac{1}{2} x^{2}+F y^{2} .
\end{array}\right.
$$

Then:

(a) If $-2 \leq F \leq 0$ the period function increases in the whole period annulus.

(b) If $4 / 3 \leq F<2$ the period function decreases in the whole period annulus.

(c) If $F=2$ the origin is an isochronous center. 
Proof. The function $g(u)$ of the corresponding potential system has the following expression:

$$
g(u)=\frac{1}{2}(1+F u)-\frac{1}{2}(1+F u)^{1-2 / F} .
$$

Its successive derivatives are

$$
\begin{aligned}
g^{\prime}(u) & =\frac{F}{2}-\frac{F-1}{2}(1+F u)^{-2 / F}, \\
g^{\prime \prime}(u) & =(F-2)(1+F u)^{-1-2 / F}, \\
g^{(3)}(u) & =-\left(F^{2}-4\right)(1+F u)^{-2-2 / F} .
\end{aligned}
$$

The associated Schaaf function is

$$
\begin{aligned}
S(g)= & 5(F-2)^{2}(1+F u)^{-2-4 / F} \\
& +3\left(F^{2}-4\right)(1+F u)^{-2-2 / F}\left[\frac{F}{2}-\frac{F-1}{2}(1+F u)^{-2 / F}\right] \\
= & {\left[5(F-2)^{2}-\frac{3}{2}\left(F^{2}-4\right)(F-2)\right](1+F u)^{-2-4 / F} } \\
& +\frac{3}{2} F\left(F^{2}-4\right)(1+F u)^{-2-2 / F} \\
= & (F-2)^{2}\left(2-\frac{3}{2} F\right)(1+F u)^{-2-4 / F}+\frac{3}{2} F\left(F^{2}-4\right)(1+F u)^{-2-2 / F} .
\end{aligned}
$$

We see that if $4 / 3 \leq F<2$ then $S(g)<0$. If $-2 \leq F \leq 0$ then $S(g)>0$. This proves the theorem.

Perhaps it is possible to extend Theorem 6 by using Rothe's function $R(g)$ instead of $S(g)$. But the calculation seems to be hard.

3.2.4. Systems $\left(L_{0, F}\right)$

TheOREM 7. Consider the system $\left(L_{0, F}\right)$ with a center at 0 ,

$$
\left(L_{0, F}\right) \quad\left\{\begin{array}{l}
\dot{x}=-y+x y \\
\dot{y}=x+F y^{2}
\end{array}\right.
$$

Then:

(a) If $2 / 3 \leq F<1$ the period function decreases in the whole period annulus.

(b) If $F=1$ the origin is an isochronous center.

Proof. The function $g(u)$ of the potential system has the following expression:

$$
g(u)=(1+F u)-(1+F u)^{1-1 / F} .
$$


Its derivatives are

$$
\begin{aligned}
g^{\prime}(u) & =F-(F-1)(1+F u)^{-1 / F}, \\
g^{\prime \prime}(u) & =(F-1)^{-1-1 / F}, \\
g^{(3)}(u) & =\left(1-F^{2}\right)^{-2-1 / F} .
\end{aligned}
$$

The corresponding Schaaf function is

$$
\begin{aligned}
S(g)= & 5(F-1)^{2}(1+F u)^{-2-2 / F} \\
& +3\left(F^{2}-1\right)(1+F u)^{-2-1 / F}\left[F-(F-1)(1+F u)^{-1 / F}\right] \\
= & {\left[5(F-1)^{2}-3\left(F^{2}-1\right)(F-1)\right](1+F u)^{-2-2 / F} } \\
& +3 F\left(F^{2}-1\right)(1+F u)^{-2-1 / F} \\
= & (F-1)^{2}(2-3 F)(1+F u)^{-2-2 / F}+3 F\left(F^{2}-1\right)(1+F u)^{-2-1 / F} .
\end{aligned}
$$

Thus $S(g)<0$ if $2 / 3 \leq F<1$.

3.2.5. Systems $\left(L_{D, 1}\right)$

TheOREM 8. Consider the system $\left(L_{D, 1}\right)$ with a center at 0 ,

$$
\left(L_{D, 1}\right)\left\{\begin{array}{l}
\dot{x}=-y+x y \\
\dot{y}=x+D x^{2}+y^{2} .
\end{array}\right.
$$

Then:

(a) If $D>0$ the period function increases in the whole period annulus.

(b) If $D<-1$ the period function increases in the whole period annulus.

(c) If $D=0$ the origin is an isochronous center.

Proof. The function $g(u)$ of the potential system has the following expression:

$$
g(u)=(1+D)(1+u)-(1+2 D)+D(1+u)^{-1} .
$$

Its derivatives are

$$
\begin{aligned}
g^{\prime}(u) & =(1+D)-D(1+u)^{-2}, \\
g^{\prime \prime}(u) & =2 D(1+u)^{-3}, \\
g^{(3)}(u) & =-6 D(1+u)^{-4} .
\end{aligned}
$$

Thus, the Schaaf function is

$$
\begin{aligned}
S(g) & =20 D^{2}(1+u)^{-6}+18 D(1+u)^{-4}\left[1+D-D(1+u)^{-2}\right] \\
& =20 D^{2}(1+u)^{-6}+18 D(D+1)(1+u)^{-4}-18 D^{2}(1+u)^{-6} \\
& =2 D^{2}(1+u)^{-6}+18 D(1+D)(1+u)^{-4} \\
& =2 D(1+u)^{-6}\left[D+9(1+D)(1+u)^{2}\right] .
\end{aligned}
$$

Hence, if $D \geq 0$ then $S(g) \geq 0$ and if $D \leq-1$ then $S(g)>0$. 
Notice that at $u=0$ one gets $S(g)(0)=2 D(10 D+9)$. This vanishes for $D=-9 / 10$. The value $D=-9 / 10$ belongs to the remaining interval $[-1,0]$, which is not covered by Theorem 8 .

Perhaps it could be possible to improve estimates of $D$ in Theorem 8 .

A calculation of the Rothe function $R(g)$ brings after simplifying

$$
\begin{aligned}
R(g)= & 2 D\left((1+u)^{-1}-1\right)\left[D^{2}(1+u)^{-3}+\left(5 D^{2}+2 D-3\right)(1+u)^{-2}\right. \\
& \left.-3(1+D)^{2}(1+u)^{-1}-3(1+D)^{2}\right] .
\end{aligned}
$$

For $D=-9 / 10$ the Rothe function becomes

$$
R(g)=-\frac{9}{5}\left[(1+u)^{-1}-1\right]^{2}\left[81(1+u)^{-2}+6(1+u)^{-1}+3\right],
$$

which also does not permit one to conclude.

On the other hand, the period function can be represented as a power series

$$
T(\xi, D, F)=2 \pi+p_{2}(D, F) \xi^{2}+p_{4}(D, F) \xi^{6}+\cdots .
$$

The Quadratic Period Coefficient Lemma (part (ii) of Lemma 3.1 of [C-J]) gives for Loud systems $\left(L_{D, F}\right)$ the first two nonzero coefficients $p_{2}, p_{4}$ :

$$
\begin{aligned}
p_{2}(D, F)= & \frac{\pi}{12}\left[10 d^{2}+10 D F-D+4 F^{2}-5 F+1\right]=\frac{\pi}{12} S(g)_{u=0}, \\
p_{4}(D, F)= & \frac{\pi}{1152}\left[1540 D^{4}+4040 D^{3} F+1180 D^{3}+4692 D^{2} F^{2}+1992 D^{2} F\right. \\
& +453 D^{2}+2768 D F^{3}+228 D F^{2}+318 D F-2 D \\
& \left.+784 F^{4}-616 F^{3}-63 F^{2}-154 F+49\right] .
\end{aligned}
$$

For $F=1$ one gets

$$
p_{4}(D, 1)=\frac{70988}{125}-\frac{44296}{25} F+\frac{88308}{25} F^{2}-\frac{15536}{5} F^{3}+784 F^{4} .
$$

When $D=-9 / 10$ one obtains

$$
p_{4}\left(-\frac{9}{10}, 1\right)=\frac{648}{125} .
$$

That means the system $\left(L_{-9 / 10,1}\right)$ has an increasing period near 0 .

4. Appendix: General Loud system. This part is useful when we wish to go further in the research of other monotonicity cases for the period function and when we are interested in the conjecture of [C-J]. The following calculations can also contribute to a certain classification of systems $\left(L_{D, F}\right)$ beyond the quadratic systems. Recall the conjecture of $[\mathrm{C}-\mathrm{J}]: \lambda_{3}=\lambda_{6}$ in the Bautin form

$$
\left(B_{\lambda}\right)\left\{\begin{array}{l}
\dot{x}=-y-\lambda_{3} x^{2}+\left(2 \lambda_{2}+\lambda_{5}\right) x y+\lambda_{3} y^{2} \\
\dot{y}=x+\lambda_{2} x^{2}+\left(2 \lambda_{3}+\lambda_{4}\right) x y-\lambda_{2} y^{2}
\end{array}\right.
$$


and if the system is not isochronous, then the period function is globally increasing.

Our results below may be viewed as a contribution to that conjecture. For $D, F \in \mathbb{R}$ we get the Loud function

$$
g(u)=(1+D)(1+F u)-(1+2 D)(1+F u)^{1-F^{-1}}+D(1+F u)^{1-2 F^{-1}} .
$$

This function permits us to transform the system (with a center at 0 )

$$
\left(L_{D, F}\right) \quad\left\{\begin{array}{l}
\dot{x}=-y+x y, \\
\dot{y}=x+D x^{2}+F y^{2},
\end{array}\right.
$$

to the potential one

$$
\frac{d u}{d t}=-y, \quad \frac{d v}{d t}=g(u)
$$

with

$$
\begin{aligned}
& x=1-(1+F u)^{-1 / F} \equiv \phi(u), \\
& y=\frac{\dot{x}}{x-1}=\frac{\phi^{\prime}(u) v}{\phi(u)-1} .
\end{aligned}
$$

This system also has a center at 0 .

The function $g$ has remarkable properties. Its derivatives are

$$
\begin{aligned}
g^{\prime}(u)= & (1+D) F-(F-1)(1+2 D)(1+F u)^{-F^{-1}} \\
& +D(-2+F)(1+F u)^{-2 F^{-1}}, \\
g^{\prime \prime}(u)= & (F-1)(1+2 D)(1+F u)^{-1-F^{-1}} \\
& -2 D(-2+F)(1+F u)^{-1-2 F^{-1}}, \\
g^{(3)}(u)= & \left(F^{2}-1\right)(1+2 D)(1+F u)^{-2-F^{-1}} \\
& +2 D\left(F^{2}-4\right)(1+F u)^{-2 F^{-1}-2}, \\
\frac{g^{(4)}(u)}{F+1}= & 2(1+F u)^{-(2 F+1) / F} F^{2}-4 D(1+F u)^{-2(F+1) / F} F^{2} \\
& +4(1+F u)^{-(2 F+1) / F} F^{2} D-(1+F u)^{-(2 F+1) / F} F \\
& -2(1+F u)^{-(2 F+1) / F} D F-(1+F u)^{-(2 F+1) / F} \\
& -2(1+F u)^{-(2 F+1) / F} D+16 D(1+F u)^{-2(F+1) / F}(1+F u)^{-1} .
\end{aligned}
$$

Notice that their values at 0 are

$$
\begin{gathered}
g^{\prime}(0)=1, \quad g^{\prime \prime}(0)=F-1+2 D, \quad g^{(3)}(0)=1-F^{2}-6 D, \\
g^{(4)}(0)=-\left(F^{2}-1\right)(1+2 D)\left(-2-F^{-1}\right) F+2 D\left(F^{2}-4\right)\left(-2-2 F^{-1}\right) F \\
=(F+1)\left(14 D-2 D F+2 F^{2}-F-1\right) .
\end{gathered}
$$

A particular property of $g$ is given by the following 


\section{LEMMA.}

(a) If $g^{\prime \prime}(0)=g^{(3)}(0)=0$ then the center 0 of the corresponding Loud system is isochronous.

(b) If $g^{(3)}(0)=g^{(4)}(0)=0$ then the period function of the corresponding system is monotonic.

(c) If $g^{\prime \prime}(0)=g^{(4)}(0)=0$ then $g=g(u)$ is an odd function and the period function of the corresponding system is monotonic.

Proof. Indeed, we may deduce from the above that

$$
\begin{aligned}
g^{\prime \prime}(0) & =F-1+2 D, \quad g^{(3)}(0)=1-F^{2}-6 D, \\
g^{(4)}(0) & =(F+1)\left(14 D-2 D F+2 F^{2}-F-1\right), \\
g^{(4)}(0) & =(F+1)[-7(F-1)+F(F-1)+(2 F+1)(F-1)] \\
& =3(F+1)(F-1)(F-2) .
\end{aligned}
$$

For case (a) one gets $F=1, D=0$. Case (b) implies $F^{2}-4=0$ and $D=-1 / 2$. For $F=2$, the center is isochronous. $F=-2$ is covered by Theorem 6.

Concerning case (c) three subcases may occur:

(i) $F=-1$ implies $D=1$.

(ii) $F=1$ implies $D=0$ and implies $g^{\prime \prime \prime}(0)=0$.

(iii) $F=2$ implies $D=-1 / 2$ and implies $g^{\prime \prime \prime}(0)=0$.

We obtain the corresponding expressions of $g$ :

$$
g(u)= \begin{cases}u(1-u)(1+u) & \text { for (i), } \\ u & \text { for (ii) and (iii), }\end{cases}
$$

which corresponds to the isochronous center established by Loud.

We now return to a general system $\left(L_{D, F}\right)$ with a center. In order to study monotonicity properties of the period function for the equation

$$
u^{\prime \prime}+g(u)=0
$$

we need to know the Schaaf and Rothe functions.

The Schaaf function

$$
S(g)=5 g^{\prime \prime 2}-3 g^{\prime} g^{(3)}
$$

has the following form:

$$
\begin{aligned}
S(g)= & 5\left((F-1)(1+2 D)(1+F u)^{-1-F^{-1}}-2 D(-2+F)(1+F u)^{-1-2 F^{-1}}\right)^{2} \\
& -3\left((1+D) F-(F-1)(1+2 D)(1+F u)^{-F^{-1}}\right. \\
& \left.+D(-2+F)(1+F u)^{-2 F^{-1}}\right)-\left(\left(F^{2}-1\right)(1+2 D)(1+F u)^{-2-F^{-1}}\right. \\
& \left.+2 D\left(F^{2}-4\right)(1+F u)^{-2 F^{-1}-2}\right) .
\end{aligned}
$$


Then after developing, simplifying and substituting $(1+F u)^{-1 / F}=x$ we obtain

$$
\begin{aligned}
(1+F u)^{2} & x^{4} S(g) \\
= & 9 F^{3} x^{3}-64 F^{2} D^{2} x-4 F D^{2} x^{2}+32 F^{2} D^{2} x^{2}-32 D F^{2} x-F D x^{2} \\
& +32 D F^{2} x^{2}+66 F D^{2} x+33 F D x+18 D^{2} F^{3} x+9 D F^{3} x-9 F D x^{3} \\
& -18 D^{2} F^{3} x^{2}-18 D F^{3} x^{2}-6 F D^{2} x^{3}+6 D^{2} F^{3} x^{3}-3 F x^{3}-3 F^{3} x^{2} \\
& +3 F^{3} x^{3}-20 D^{2} x-7 F x^{2}+8 F^{2} x^{2}+8 D^{2} x^{2}-10 D x+8 D x^{2} \\
& -56 F D^{2}-6 D^{2} F^{3}+32 F^{2} D^{2}+2 x^{2}+32 D^{2} .
\end{aligned}
$$

Write now

$$
(1+F u)^{2} x^{4} S(g)=v_{3} x^{3}+v_{2} x^{2}+v_{1} x+v_{0} .
$$

Then

$$
\begin{aligned}
v_{3}= & 3 F^{3}-9 F D+9 D F^{3}-6 F D^{2}+6 D^{2} F^{3}-3 F \\
= & 3 F(F-1)(F+1)(1+D)(1+2 D), \\
v_{2}= & -4 F D-18 D F^{3}-4 F D^{2}+8 D+32 F^{2} D^{2}-3 F^{3}+8 D^{2}+2 \\
& -7 F+8 F^{2}+32 D F^{2}-18 D^{2} F^{3} \\
= & \left(-4 F+32 F^{2}+8-18 F^{3}\right) D^{2}+\left(-4 F+32 F^{2}+8-18 F^{3}\right) D \\
& -7 F-3 F^{3}+2+8 F^{2} \\
= & \left(-4 F+32 F^{2}+8-18 F^{3}\right) D^{2}+\left(-4 F+32 F^{2}+8-18 F^{3}\right) D \\
& -(3 F-2)(F-1)^{2}, \\
v_{1}= & -20 D^{2}+18 D^{2} F^{3}+9 D F^{3}+66 F D^{2}-32 D F^{2} \\
& -64 F^{2} D^{2}-10 D+33 F D \\
= & D(9 F-5)(F-1)(-2+F)(1+2 D), \\
v_{0}= & -6 D^{2} F^{3}+32 F^{2} D^{2}+32 D^{2}-56 F D^{2} \\
= & -2 D^{2}(-4+3 F)(-2+F)^{2} .
\end{aligned}
$$

Moreover, one gets

$$
\begin{aligned}
S(g)_{u=0} & =5 g^{\prime \prime}(0)-3 g^{\prime}(0) g^{(3)}(0)=v_{0}+v_{1}+v_{2}+v_{3} \\
& =8 F^{2}-10 F+20 F D+2-2 D+20 D^{2} .
\end{aligned}
$$

The Rothe function is

$$
\begin{aligned}
R(g) & =3 g^{\prime \prime}(0) g^{\prime}(u)^{2}-g^{\prime \prime}(0) g(u) g^{\prime \prime}(u)-3 g^{\prime \prime}(u) \\
& =3(F-1+2 D) g^{\prime}(u)^{2}-(F-1+2 D) g(u) g^{\prime \prime}(u)-3 g^{\prime \prime}(u)
\end{aligned}
$$




$$
\begin{aligned}
= & (F-1+2 D)\left[3\left(F^{2}+2 F^{2} D+F^{2} D^{2}\right) x^{4}\right. \\
& +3\left(-6 F^{2} D+6 D F+2 F-2 F^{2}+4 F D^{2}-4 F^{2} D^{2}\right) x^{3} \\
& +3\left(-2 F-12 D F+F^{2}+4 D+6 F^{2} D^{2}+6 F^{2} D+1+4 D^{2}-12 F D^{2}\right) x^{2} \\
& +3\left(6 D F-8 D^{2}+12 F D^{2}-4 F^{2} D^{2}-2 F^{2} D-4 D\right) x-10 F D^{2}+3 F^{2} D^{2} \\
& +8 D^{2}-\left(-1+3 D F+F-3 D-2 D^{2}+2 F D^{2}\right) x^{3}-\left(8 D-6 F D^{2}\right. \\
& \left.\left.+8 D^{2}-F-6 D F+1\right) x^{2}-\left(3 D F-5 D-10 D^{2}+6 F D^{2}\right) x\right] \\
& -3(F-1)(1+2 D)(1+F u)^{-1-1 / F}+6 D(F-2)(1+F u)^{-1-2 / F},
\end{aligned}
$$

where $(1+F u)^{-1 / F}=x$. We now write

$$
\begin{aligned}
R(g)= & a_{4} x^{4}+a_{3} x^{3}+a_{2} x^{2}+a_{1} x+a_{0} \\
& -3(F-1)(1+2 D)(1+F u)^{-1-1 / F}+6 D(F-2)(1+F u)^{-1-2 / F},
\end{aligned}
$$

where the coefficients are

$$
\begin{aligned}
a_{4}= & (F-1+2 D)\left(3 F^{2}+6 F^{2} D+3 F^{2} D^{2}\right)=3(F-1+2 D) F^{2}(1+D)^{2}, \\
a_{3}= & (F-1+2 D)\left(1+15 D F+5 F+3 D+2 D^{2}+10 F D^{2}\right. \\
& \left.-18 F^{2} D-6 F^{2}-12 F^{2} D^{2}\right) \\
= & -(F-1+2 D)(6 F+1)(F-1)(1+D)(1+2 D), \\
a_{2}= & (F-1+2 D)\left(4 D-30 F D^{2}+4 D^{2}-5 F-30 D F+2+3 F^{2}\right. \\
& \left.+18 F^{2} D^{2}+18 F^{2} D\right) \\
= & (F-1+2 D)\left[2 D(D+1)\left(9 F^{2}-15 F+2\right)+(F-1)(3 F+5)\right], \\
a_{1}= & (F-1+2 D)\left(15 D F-14 D^{2}+30 F D^{2}-12 F^{2} D^{2}-6 F^{2} D-7 D\right) \\
= & -(F-1+2 D) D\left(7-15 F+6 F^{2}\right)(1+2 D), \\
a_{0}= & (F-1+2 D)\left(-10 F D^{2}+3 F^{2} D^{2}+8 D^{2}\right) \\
= & (F-1+2 D) D^{2}(3 F-4)(F-2) .
\end{aligned}
$$

Acknowledgements. I would like to thank Jean-Marie Strelcyn for fruitful discussions and the referee for many valuable remarks.

\section{References}

[C-S] J. Chavarriga and M. Sabatini, A survey of isochronous centres, Qual. Theory Dynam. Systems 1 (1999), 1-70.

[C] C. Chicone, Geometric methods for two-point nonlinear boundary value problems, J. Differential Equations 72 (1988), 360-407.

[C-D] C. Chicone and F. Dumortier, A quadratic system with a nonmonotonic period function, Proc. Amer. Math. Soc. 102 (1988), 706-710. 
[C-J] C. Chicone and M. Jacobs, Bifurcation of critical periods for plane vector fields, Trans. Amer. Math. Soc. 312 (1989), 433-486.

[Ch-C] R. Chouikha and F. Cuvelier, Remarks on some monotonicity conditions for the period function, Appl. Math. (Warsaw) 26 (1999), 243-252.

[C-W] S. N. Chow and D. Wang, On the monotonicity of the period function of some second order equations, Casopis Pěst. Mat. 111 (1986), 14-25.

[CGM] A. Cima, A. Gasull and F. Mañosas, Period function of a class of Hamiltonian systems, J. Differential Equations 168 (2000), 180-199.

[CLY] S. N. Chow, C. Z. Li and Y. F. Yi, The cyclicity of period annulus of degenerate quadratic Hamiltonian systems with elliptic segment, Ergodic Theory Dynam. Systems 22 (2002), 347-349.

[F-G-G] E. Freire, A. Gasull and A. Guillamon, First derivative of the period function with applications, J. Differential Equations 204 (2004), 139-162.

[L] W. S. Loud, The behavior of the period of solutions of certain plane autonomous systems near centers, Contr. Differential Equations 3 (1964), 21-36.

[O] Z. Opial, Sur les périodes des solutions de l'équation différentielle $x^{\prime \prime}+g(x)=0$, Ann. Polon. Math. 10 (1961), 49-72.

[RSC] R. Reissig, G. Sansone and R. Conti, Qualitative Theorie nichtlinearer Differentialgleichungen, Ed. Cremonese, Roma, 1963.

[R] F. Rothe, Remarks on periods of planar Hamiltonian systems, SIAM J. Math. Anal. 24 (1993), 129-154.

[Sc] R. Schaaf, A class of Hamiltonian systems with increasing periods, J. Reine Angew. Math. 363 (1985), 96-109.

LAGA

Université Paris 13

93430 Villetaneuse, France

E-mail: chouikha@math.univ-paris13.fr

Received on 9.11.2004;

revised version on 19.9.2005 